BLADDER CANCER

\section{Chemotherapy and checkpoint blockade}

A phase II trial of gemcitabine and cisplatin (GC) plus ipilimumab in 36 chemotherapy-naive patients with metastatic urothelial cancer did not achieve its primary end point of $>60 \%$ 1 -year overall survival. Further analysis showed that circulating $\mathrm{CD}^{+}{ }^{+} \mathrm{T}$ cells expanded significantly following addition of ipilimumab to GC, which correlated with improved survival. Furthermore, the response rate was significantly higher in patients with deleterious somatic DNA damage response (DDR) gene mutations.

"We designed this study based on early findings of CTLA4 inhibition and before data on PD1-PDL1 blockade in urothelial cancer were available," explains lead author Matthew Galsky from the Icahn School of Medicine at Mount Sinai in New York, USA. "Whether CTLA4 blockade alone would be effective was unclear, so we decided to exploit a possible 'autovaccination' effect caused by chemotherapy, which might combine favourably with CTLA4 blockade." Using a phased treatment schedule (two cycles of GC followed by four cycles of GC plus ipilimumab), the team evaluated whether immunosuppressive effects of chemotherapy might hamper its immunomodulatory effects, and whether responses depend on a specific genomic tumour make up. "We hypothesized that tumours with DDR gene mutations might be particularly sensitive to the combination of cisplatin-based chemotherapy plus immune checkpoint blockade, owing to data indicating a beneficial effect of such alterations on the activity of either treatment alone," summarizes Galsky.

The trial data show that chemotherapy plus CTLA4 blockade can be safely administered in patients with metastatic urothelial cancer, similar to other immune checkpoint inhibitors. Grade $\geq 3$ adverse events (predominantly haematological) occurred in $81 \%$ of patients. Peripheral blood immune cell analysis showed an expansion of T cells following ipilimumab in some patients, which was associated with extended survival, suggesting that chemotherapy does not limit the activity of immune checkpoint blockade. Deleterious tumour DDR gene mutations were associated with increased total mutational burden and a significantly increased likelihood of response $(P=0.03)$; ten patients with these alterations had a partial or complete response. Testing whether $\mathrm{GC}$ induces immunogenic cell death, the researchers found no increase in levels of HMGB1, a danger-associated signal molecule, after two cycles of GC.

"We will soon launch two studies using PD1 blockade that build on these results," Galsky concludes. "The first study tests whether patients with DDR mutations might be particularly sensitive to treatment with cisplatin-based chemotherapy plus immune checkpoint blockade. The second study evaluates whether specific chemotherapy agents are preferable for immunotherapy-based combinations."

Clemens Thoma

ORIGINAL ARTICLE Galsky, M. D. et al. Phase 2 trial of gemcitabine, cisplatin, plus ipilimumab in patients with metastatic urothelial cancer and impact of DNA damage response gene mutations on outcomes. Eur. Urol. http://dx.doi.org/ 10.1016/j.eururo.2017.12.001 (2017) 